Sains Malaysiana 49(4)(2020): 919-928

http://dx.doi.org/10.17576/jsm-2020-4904-21

\title{
Limit Theorem for A Semi-Markovian Random Walk with General Interference of Chance
}

(Had Teorem untuk Jalan Rawak Semi-Markovan dengan Kemungkinan Gangguan Umum)

\author{
TAHIR KHANIYEV \& OZlEM ARDiC SEVINC*
}

\begin{abstract}
A semi-Markovian random-walk process with general interference of chance was constructed and investigated. The key point of this study is the assumption that the discrete interference of chance has a general form. Under some conditions, it is proved that the process is ergodic, and the exact forms of the ergodic distribution and characteristic function of the process are obtained. By using basic identity for random walks, the characteristic function of the process is expressed by the characteristic function of a boundary functional. Then, two-term asymptotic expansion for the characteristic function of the standardized process is found. Using this asymptotic expansion, a weak convergence theorem for the ergodic distribution of the standardized process is proved, and the limiting form for the ergodic distribution is obtained. The obtained limit distribution coincides with the limit distribution of the residual waiting time of the renewal process generated by a sequence of random variables expressing the discrete interference of chance.
\end{abstract}

Keywords: Discrete interference of chance; ergodic distribution; limit distribution; random walk; weak convergence

ABSTRAK

Proses jalan rawak semi-Markovan dengan kemungkinan gangguan umum telah dibangunkan dan dikaji. Isi utama kajian ini adalah andaian bahawa kemungkinan gangguan diskrit mempunyai bentuk umum. Dalam beberapa keadaan, terbukti bahawa prosesnya ergodik dan bentuk asal taburan ergodik serta fungsi pencirian prosesnya diperoleh. Dengan menggunakan identiti asas untuk jalan rawak, fungsi pencirian prosesnya diungkapkan oleh fungsi pencirian sempadan fungsian. Kemudian, pengembangan asimptotik dua penggal untuk fungsi pencirian piawai prosesnya ditemui. Dengan menggunakan pengembangan asimtotik ini, teorem penumpuan yang lemah untuk taburan ergodik daripada proses piawai dibuktikan dan bentuk pembatasan untuk taburan ergodik diperoleh. Taburan had yang diperoleh bertepatan dengan had taburan sisa masa menunggu proses pembaharuan yang dihasilkan oleh jujukan pemboleh ubah rawak yang mengungkapkan kemungkinan gangguan diskrit.

Kata kunci: Had taburan; jalan rawak; kemungkinan gangguan diskrit; penumpuan yang lemah; taburan ergodik

\section{INTRODUCTION}

Renewal, renewal reward, random-walk, Markov, and semi-Markov processes provide a natural means of modeling real systems of stock control, mathematical insurance, queuing theory, mathematical biology, reliability, stochastic risk, physics, and other theories. These processes have been studied by several authors (Aliyev et al. 2009; Alsmeyer 1991; Başar 2017; Brown \& Solomon 1975; Chang \& Peres 1997; Feller 1971; Gihman \& Skorokhod 1975; Gökpınar et al. 2015; Hanalioglu \& Khaniyev 2019; Janseen \& Leeuwarden 2007; Khaniyev \& Mammadova 2006; Lotov 1996; Samsuddin \& Ismail 2019; Siegmund 1979). In the earlier reports, the treatment was purely analytical and theoretical, while the later studies adopted an approximation approach.

Studies on these processes mainly focus on such subjects as renewal function, maximum of a random walk, limiting behavior, ladder heights, boundary functionals, and ergodicity. The renewal function $U(t)$ plays a key role in semi-Markovian random-walk processes. A decomposition with respect to the jump value, the random time of the first renewal, yields an integral equation of a type known as renewal equations (Feller 1971), while Brown and Solomon (1975) derived expansion for variance of $N(t)$ as $t$ $\rightarrow \infty$, where $N(t)$ is a renewal process. However, Alsmeyer (1991) investigated the harmonic renewal function and provided an interesting probabilistic alternative. Investigation of the limiting behavior of semi-Markov processes is one of the central problems in the theory of stochastic processes. When speaking of convergence of the distributions of the random variables $X_{n}, n=1,2, \ldots$, to the distribution of the random variable $X$, in probability theory, one usually means weak convergence. Feller (1971) considered limit theorems for the number of times the simplest kind of random walk hits a certain state. Gihman and Skorohod (1975) proved a general ergodic theorem of processes, including semi-Markov processes. Consider the 
random walk $\left\{S_{n}\right\}, n \geq 0$ consisting of partial sums $S_{n}=\eta_{1}$ $+\ldots+\eta_{n}$. The first time $v_{1}^{+}=\min \left\{\mathrm{n} \geq 1: S_{n}>0\right\}$ that the random walk is positive is the first ladder epoch, and the first positive value $\chi_{1}^{+}=\sum_{i=1}^{v_{1}^{+}} \eta_{i}$ taken by the random walk is the first ladder height. The first ladder height plays a fundamental role in the theory and applications of random walks (for example see Feller (1971), Rogozin (1964), and Siegmund (1979)). In the studies of Lotov (1996), Chang and Peres (1997), and Nagaev (2010), exact and obvious expressions were derived for the first three, four, and five moments of ladder height of the Gaussian random walk, respectively. Janseen and Leewardeen (2007) presented explicit expressions for the mean and variance of the maximum $M=\max \left\{S_{n}: n \geq 0\right\}$ of random walk, which are in terms of the Taylor series about $\beta=0$ with coefficients that involve the Riemann zeta function, while Gökpınar et al. (2013) found the approximation formulas for the first four moments of the maximum of a Gaussian random walk for the parameter $\beta \in(0.5,3.2]$ using metamodeling. Here, parameter $\beta$ is the mean of summands of the random walk $\left\{S_{n}\right\}$. Blanchet and Glynn (2006) proposed a method to compute coefficients in the asymptotic expansion of the moments of the first ladder heights for non-Gaussian random walks.

In this study, a semi-Markovian random-walk process with general interference of chance is considered. This process belongs to a wide class of stochastic processes, which is known as 'the class of the semi-Markov processes with a discrete interference of chance'. This class of stochastic processes was first introduced by Kolmogorov in the 1960s and 1970s. To have a broad idea about random-walk processes with discrete interference of chance, it is necessary to review the literature. Khaniyev and Mammadova (2006) investigated an extended model of type $(s, S)$ with Gaussian distribution of summands and obtained results for the stationary characteristics of the model. In addition, Khaniyev et al. (2008) found asymptotic expansions for the ergodic moments when the discrete interference of chance has an exponential distribution. Aliyev et al. $(2010,2009)$ and Kesemen et al. (2013) obtained results when the discrete interference of chances has gamma, triangular, and Weibull distributions. Hanalioglu et al. (2015) investigated a random-walk process with normal distributed interference of chance and proved a weak convergence theorem. In the studies conducted so far, the random variable $\zeta_{n}$ corresponding to the discrete interference of chance has specific distributions. Contrary to the aforementioned studies, in the present study, results are obtained for the case where the discrete interference of chance is chosen from a wider class. In this case, this study becomes a generalization of the relevant studies so far.

The rest of this work is designed as follows. In the next section, the mathematical construction of the process $X(t)$ is presented. Then, the ergodicity of the process $X(t)$ and exact expressions for the ergodic distribution function and characteristic function of the process $X(t)$ are given.
After that, a weak convergence theorem for the ergodic distribution is proved, and the limiting form of the ergodic distribution is obtained. Finally, conclusions are drawn. Now, the process $X(t)$ is constructed mathematically.

\section{MATHEMATICAL CONSTRUCTION OF THE PROCESS $X(t)$}

Let $\left\{\xi_{n}\right\},\left\{\eta_{n}\right\}$, and $\left\{\zeta_{n}\right\}, n \geq 1$ be three independent sequences of random variables defined on a probability space $(\Omega, F, P)$, such that variables in each sequence are independent and identically distributed. Suppose that random variables $\xi_{n}$ and $\zeta_{n}$ take positive values, while random variable $\eta_{n}$ takes positive and negative values. Denote the distribution functions of $\xi_{n}, \eta_{n}$, and $\zeta_{n}$ by $\Phi(\mathrm{t}), F(x)$, and $\pi(z)$, respectively, i.e.,

$$
\begin{gathered}
\Phi(t)=P\left\{\xi_{1} \leq t\right\} ; F(x)=P\left\{\eta_{1} \leq x\right\} ; \\
\pi(z)=P\left\{\zeta_{1} \leq \mathrm{z}\right\} ; \mathrm{t} \geq 0 ; z>0 ; x \in(-\infty, \infty) .
\end{gathered}
$$

Define renewal sequence $\left\{T_{n}\right\}$ and random walk $\left\{S_{n}\right\}$ as

$$
T_{n}=\sum_{i=1}^{n} \xi_{i} ; S_{n}=\sum_{i=1}^{n} \eta_{i} ; T_{0}=S_{0}=0 ; n=1,2, \ldots
$$

and a sequence of integer valued random variables $\left\{N_{n}\right\}$, $n \geq 0$ as

$N_{0}=0 ; N_{1}=N(\lambda z)=\inf \left\{\mathrm{n} \geq 1: \lambda z-S_{k}>0, k=1, n-1 ; \lambda z-\right.$ $\left.S_{n} \leq 0\right\}$

$$
\begin{gathered}
N_{m+1}=N_{m+1}\left(\lambda \zeta_{m}\right)=\inf \left\{n \geq 1: \lambda \zeta_{m}-\left(S_{L_{m}+k}-S_{L_{m}}\right)>0\right. \\
\left.k=\overline{1, n-1} ; \lambda \zeta_{m}-\left(S_{L_{m}+n}-S_{L_{m}}\right) \leq 0\right\}
\end{gathered}
$$

Here, $L_{\mathrm{m}}=N_{1}+N_{2}+\ldots+N_{m}, m=1,2, \ldots$ and $\inf (\phi)=+\infty$ is stipulated. Put

$\tau_{0}=0 ; \tau_{1}=\tau_{1}(\lambda z)=T_{N(\lambda z)}=\sum_{i=1}^{N(\lambda z)} \xi_{i} ; \tau_{m}=T_{L_{m}}=\sum_{i=1}^{L_{m}} \xi_{i} ; m=1,2, \ldots$

Further, set $v(t)=\max \left\{n \geq 0: T_{n} \leq t\right\}, t>0$. Now, one can construct the desired stochastic process $X(t)$ as follows:

$X(t)=\lambda \zeta_{m-1}-\left(S_{v(t)}-S_{L_{m-1}}\right)$, when $\tau_{m-1} \leq t<\tau_{m} ; m=1,2, \ldots$

Here, $S_{v\left(\tau_{m}\right)}=S_{N_{m}}, \zeta_{0}=z, \lambda>0$.

In this study, the random variable $\zeta_{n}$ corresponding to the discrete interference of chance can take an arbitrary distribution, so the process $X(t)$ is called a 'semiMarkovian random-walk process with general interference of chance'. A sample path of the process $X(t)$ is given in Figure 1. 


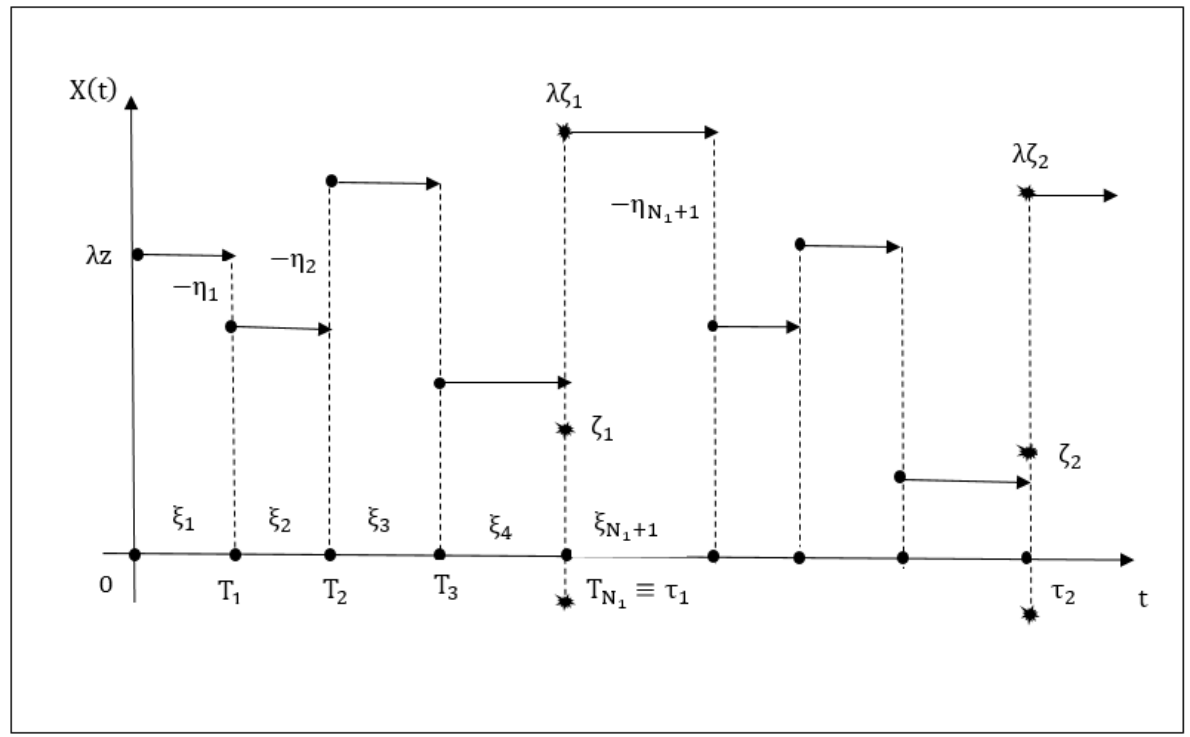

FIGURE 1. Sample path of the process $X(t)$

The main purpose of this study was to prove a weak convergence theorem for the ergodic distribution of the process $X(t)$, as $\lambda \rightarrow \infty$ when the discrete interference of chance has a general form, and to obtain the limiting form of the ergodic distribution.

\section{ERGODICITY OF THE PROCESS $X(t)$}

Calculating the finite dimensional distributions of the process $X(t)$ has computational complexity because of its mathematical structure, such as $n$-fold integrals and series. To overcome this complexity, it is useful to calculate the stationary characteristics of the process $X(t)$. For this purpose, it is first shown that the process $X(t)$ is ergodic under certain conditions. To achieve this, the following lemma is necessary.

Lemma 1 (Aliyev et al. 2016). Let $\mathrm{g}: R^{+} \rightarrow R$ be a bounded and measurable function and $\sup _{\mathrm{x}}|\mathrm{g}(\mathrm{x})| \equiv \mathrm{H}<\infty$ Addititionally, $\lim _{\mathrm{x} \rightarrow \infty} \mathrm{g}(\mathrm{x})=0$ and $\pi(0) \equiv \mathrm{P}\left\{\zeta_{1} \leq 0\right\}=0$. Then, the following asymptotic relation holds, as $\lambda \rightarrow \infty$ :

$$
\int_{z=0}^{\infty} g(\lambda z) d \pi(z) \rightarrow 0 .
$$

Here, $\pi(z)=P\left\{\zeta_{1} \leq z\right\}, z>0$.

Now, the ergodicity of the process $X(t)$ is stated in the following theorem.

Theorem 1 Let the initial sequences of random variables $\left\{\xi_{n}\right\},\left\{\eta_{n}\right\}$, and $\left\{\zeta_{n}\right\}, \mathrm{n} \geq 1$, satisfy the following supplementary conditions:

i) $0<E\left(\xi_{1}\right)<\infty$; ii) $E\left(\eta_{1}\right)>0$; iii) $E\left(\eta_{1}^{2}\right)<\infty$;

iv) $\eta_{1}$ is a nonarithmetic random variable; and

v) $0<E\left(\zeta_{1}\right)<\infty$.
Then, the process $X(t)$ is ergodic.

Proof The process $X(t)$ is included in a wide class of stochastic processes called 'semi-Markov processes with a discrete interference of chance'. This class was first defined by Kolmogorov, and various aspects of this class have been investigated by numerous researchers. A general ergodic theorem for this class of processes was given by Gihman and Skorohod (1975). According to Gihman and Skorohod (1975, p. 243) study, to show the ergodicity of the process $X(t)$, it is sufficient to verify the following two assumptions.

Assumption 1 It is necessary to choose a sequence of ascending random times, such that the values of the process $X(t)$ at these times form an embedded Markov chain that is ergodic. On behalf of the random times mentioned, the sequence $\left\{\tau_{n}\right\}, \mathrm{n} \geq 0$ defined in the mathematical construction in the process $X(t)$ section can be chosen. By definition, $0=\tau_{0}<\tau_{1}<\tau_{2}<\ldots<\tau_{n}<$ $\tau_{n+1}<\ldots<\infty$. The values that the process $X(t)$ takes at these times, such as $\left(X\left(\tau_{1}\right)=\lambda \zeta_{1} ; X\left(\tau_{2}\right)=\lambda \zeta_{2} ; \ldots ; X\left(\tau_{\mathrm{n}}\right)\right.$ $\left.=\lambda \zeta_{\mathrm{n}} ; \ldots\right)$, form an ergodic Markov chain, because $\left\{\zeta_{\mathrm{n}}\right\}$, $n=1,2, \ldots$ are independent and identically distributed random variables. Therefore, $\left\{X\left(\tau_{n}\right)\right\}$ forms an ergodic Markov chain. Hence, Assumption 1 holds.

Assumption 2 The expected value of the times between consecutive random epochs $\left\{\tau_{n}\right\}, n=1,2,3, \ldots$ should be finite, i.e., for all $n=1,2,3, \ldots$

$$
\mathrm{E}\left(\tau_{n}-\tau_{n-1}\right)<\infty, n=1,2,3 \ldots
$$

Because $\tau_{n}-\tau_{n-1}, n=2,3, \ldots$ are independent and identically distributed random variables, it is sufficient to show that

$E\left(\tau_{1}(\lambda z)\right)<\infty ; E\left(\tau_{n}-\tau_{n-1}\right) \equiv \int_{0}^{\infty} E\left(\tau_{1}(\lambda z)\right) d \pi(z), n=2,3, \ldots$ 
Using Wald identity (Feller 1971),

$$
E\left(\tau_{1}\right) \equiv E\left(\tau_{1}(\lambda z)\right)=E\left(\sum_{i=1}^{N(\lambda z)} \xi_{i}\right)=E\left(\xi_{1}\right) E(N(\lambda z)) .
$$

In addition,

$E\left(\tau_{n}-\tau_{n-1}\right) \equiv E\left(\tau_{1}\left(\lambda \zeta_{1}\right)\right)=E\left(\xi_{1}\right) \int_{0}^{\infty} E(N(\lambda z)) d \pi(z), n=2,3, \ldots$

It is known that $E\left(\xi_{1}\right)<\infty$ according to conditions of Theorem 1 . To satisfy the conditions in (1), the following inequalities should hold:

$$
E(N(\lambda z))<\infty ; \int_{0}^{\infty} E(N(\lambda z)) d \pi(z)<\infty .
$$

For this aim, the ladder epoch $v_{1}{ }^{+}$and ladder height $\chi_{1}{ }^{+}$of the random walk $\left\{S_{n}\right\}, n \geq 0$ are introduced as

$$
v_{1}^{+}=\min \left\{n \geq 1: S_{n}>0\right\} ; \chi_{1}^{+}=S_{v_{1}^{+}}=\sum_{i=1}^{v_{1}^{+}} \eta_{i} \text {. }
$$

The pairs of random variables $\left\{\left(v_{n}^{+} ; \chi_{n}^{+}\right), \mathrm{n}=2,3, \ldots\right\}$ are mutually independent and have the same distributions with the pair of random variables $\left(v_{1}^{+} ; \chi_{1}^{+}\right)$(Feller 1971). With the help of these definitions, the boundary functionals $N(\lambda z)$ and $S_{N(\lambda z)}$ can be given according to the Dynkin principle (Rogozin 1964) as follows:

$$
N(\lambda z)=\sum_{i=1}^{H(\lambda z)} v_{i}^{+} ; S_{N(\lambda z)}=\sum_{i=1}^{H(\lambda z)} \chi_{i}^{+} .
$$

Here, $H(\lambda z)=\min \left\{n \geq 1: \sum_{i=1}^{n} \chi_{i}^{+}>\lambda z\right\}, z>0 . H(x)$ is renewal process generated by random variables $\left\{\chi_{i}^{+}\right\}$, $i \geq 1$.

By using Wald's identity,

$E(N(\lambda z))=E\left(\sum_{i=1}^{H(\lambda z)} v_{i}^{+}\right)=E\left(v_{1}^{+}\right) E(H(\lambda z))=E\left(v_{1}^{+}\right) U_{+}(\lambda z)$.

Here, $U(\lambda z) \equiv E(H(\lambda z))$ is a renewal function generated by random variables $\left\{\chi_{i}^{+}\right\}, \mathrm{n} \geq 1$. For each finite $z, E$ $(H(\lambda \mathrm{z})) \equiv U_{+}(\lambda \mathrm{z})$ is finite (Feller 1971, p. 359). Under the condition $E\left(\eta_{1}\right)>0, E\left(v_{1}^{+}\right)<\infty$. Therefore, the inequality $E(N(\lambda z))<\infty$ holds. Consequently, to prove the second inequality in (2), one should show that the following inequality holds:

$$
E\left(U_{+}\left(\lambda \zeta_{1}\right)\right) \equiv \int_{0}^{\infty} U_{+}(\lambda z) d \pi(z)<\infty .
$$

Under the assumption $\mu_{2} \equiv E\left(\chi_{1}^{+2}\right)<\infty$, according to the renewal theorem (Feller 1971, p. 366), the expansion of the renewal function $U_{+}(\lambda z)$ can be represented as follows, when $\lambda \rightarrow \infty$ :

$$
U_{+}(\lambda z)=\frac{\lambda z}{\mu_{1}}+\frac{\mu_{2}}{2 \mu_{1}^{2}}+g(\lambda z)
$$

Here, the function $\mathrm{g}(\mathrm{x})$ is defined as in Lemma 1. Substituting (4) into (3),

$$
\begin{gathered}
E\left(U_{+}\left(\lambda \zeta_{1}\right)\right) \equiv \int_{0}^{\infty} U_{+}(\lambda z) \mathrm{d} \pi(\mathrm{z})= \\
=\int_{0}^{\infty} \frac{\lambda z}{\mu_{1}} \mathrm{~d} \pi(\mathrm{z})+\int_{0}^{\infty} \frac{\mu_{2}}{2 \mu_{1}^{2}} \mathrm{~d} \pi(\mathrm{z})+\int_{0}^{\infty} g(\lambda z) \mathrm{d} \pi(\mathrm{z}) .
\end{gathered}
$$

By using Lemma 1, one obtains $\int_{0}^{\infty} g(\lambda z) d \pi(z)=o(1)$, when $\lambda \rightarrow \infty$. Then, asymptotic expansion for $E\left(U_{+}\left(\lambda \zeta_{1}\right)\right)$ is as follows, when $\lambda \rightarrow \infty$ :

$$
E\left(U_{+}\left(\lambda \zeta_{1}\right)\right)=\frac{\lambda \beta_{1}}{\mu_{1}}+\frac{\mu_{2}}{2 \mu_{1}^{2}}+o(1) .
$$

Under the condition of the present theorem, $\beta_{1} \equiv E\left(\zeta_{1}\right)<\infty$ is satisfied. Then, $E\left(U_{+}\left(\lambda \zeta_{1}\right)\right)<\infty$. As a result, $E\left(\tau_{1}\right)<\infty$ and $\left.E\left(\tau_{n}-\tau_{\mathrm{n}-1}\right)\right)<\infty, n=2,3, \ldots$

Thus, Assumption 2 holds. Because two assumptions are provided, the general ergodic theorem has been proved. Therefore, it is considered that process $X(t)$ is ergodic. This concludes the proof of Theorem 1 .

Theorem 2. Assume that the assumptions of Theorem 1 are satisfied. Then, for each measurable bounded function $f(x)(f:[0, \infty) \rightarrow \mathrm{R})$, the following relation is true with probability 1 .

$\lim _{t \rightarrow \infty} \frac{1}{t} \int_{0}^{t} f(X(u)) d u=\frac{1}{E\left(N\left(\lambda \zeta_{1}\right)\right)} \int_{0}^{\infty} f(x) d_{x}\left(E\left(A\left(x, \lambda \zeta_{1}\right)\right)\right)$,

where

$$
E\left(N\left(\lambda \zeta_{1}\right)\right)=\int_{0}^{\infty} E(N(\lambda z)) d \pi(z)
$$

$$
E\left(A\left(x, \lambda \zeta_{1}\right)\right)=\int_{0}^{\infty} A(x, \lambda z) d \pi(z) ; A(x, \lambda z)=\sum_{n=0}^{\infty} a_{n}(x, \lambda z) ;
$$

$a_{n}(x, \lambda z)=P\left\{\lambda z-S_{i}>0, i=\overline{1, n} ; \lambda z-S_{n} \leq x\right\} ; n \geq 1 ; x, z>0$.

Proof According to the general ergodic theorem (Gihman \& Skorohod 1975, p. 243) for a semi-Markov process with a discrete interference of chance, the following equation is provided with probability 1 for each measurable bounded function $f(x)$ :

$$
\begin{gathered}
\lim _{t \rightarrow \infty} \frac{1}{t} \int_{0}^{t} f(X(u)) d u=S_{f} \\
\equiv \frac{1}{E\left(\tau_{1}\right)} \int_{0}^{\infty} \int_{0}^{\infty} \int_{0}^{\infty} f(x) d_{x} G(t, x, \lambda z) d t d \pi(z),
\end{gathered}
$$

where $G(t, x, \lambda z) \equiv P_{\lambda z}\left\{\tau_{1}>t ; X(t) \leq x\right\} \equiv P\left\{\tau_{1}>t ; X\right.$ $(t) \leq x \mid X(0)=\lambda z\}$ and the calculation for $G(t, x, \lambda z)$ is as follows: 


$$
\begin{gathered}
G(t, x, \lambda z)=\sum_{n=0}^{\infty} P\left\{v(t)=n ; T_{N(\lambda z)}>t ; X(t) \leq x\right\} \\
=\sum_{n=0}^{\infty} P\left\{T_{n} \leq t<T_{n+1} ; T_{N(\lambda z)}>t ; X(t) \leq x\right\} \\
=\sum_{n=0}^{\infty} P\left\{T_{n} \leq t<T_{n+1} ; N(\lambda z)>n ; T_{N(\lambda z)}>t ; X(t) \leq x\right\} \\
=\sum_{n=0}^{\infty} P\left\{T_{n} \leq t<T_{n+1} ; N(\lambda z)>n ; \lambda z-S_{n} \leq x\right\} \\
=\sum_{n=0}^{\infty} P\left\{T_{n} \leq t<T_{n+1} ; \lambda z-S_{i}>0, i=\overline{1, n} ; \lambda z-S_{n} \leq x\right\} \\
=\sum_{n=0}^{\infty} P\left\{T_{n} \leq t<T_{n+1}\right\} P\left\{\lambda z-S_{i}>0, i=\overline{1, n} ; \lambda z-S_{n} \leq x\right\} \\
=\sum_{n=0}^{\infty}\left(\Phi^{* n}(t)-\Phi^{*(n+1)}(t)\right) a_{n}(x, \lambda z), \quad(6)
\end{gathered}
$$

where

$a_{n}(x, \lambda z)=P\left\{\lambda z-S_{i}>0, i=\overline{1, n} ; \lambda z-S_{n} \leq x\right\} ; a_{0}(x, \lambda z)=$ $\varepsilon(x-\lambda z)$;

$\Phi^{*_{n}}(t)=P\left\{T_{\mathrm{n}} \leq t\right\} ; \Phi^{* 0}(t)=\varepsilon(t)=\left\{\begin{array}{ll}1, & t \geq 0 \\ 0, & t<0\end{array} \mathrm{n}=1,2, \ldots\right.$

When the Laplace transform with respect to parameter $t$ is applied to (6), the following equation is obtained:

$$
\tilde{G}(\gamma, x, \lambda z)=\frac{1-\varphi(\gamma)}{\gamma} \sum_{n=0}^{\infty}(\varphi(\gamma))^{n} a_{n}(x, \lambda z), \gamma>0
$$

Here $\widetilde{G}(\gamma, x, \lambda z)=\int_{0}^{\infty} e^{-\gamma t} G(t, x, \lambda z) d t$ and $\varphi(\gamma) \equiv E\left(e^{-\gamma \xi_{1}}\right)$, $(\gamma>0)$.

Note that $\widetilde{G}(\gamma, x, \lambda z)$ is the Laplace transformation of $\mathrm{G}(t, x, \lambda z)$, and $\varphi(\gamma)$ is the Laplace-Stieltjes transformation of the distribution function $\Phi(t)$.

Because $\mathrm{E}\left(\xi_{1}\right)<\infty, \lim _{\gamma \rightarrow 0}(1-\varphi(\gamma)) / \gamma=E\left(\xi_{1}\right)$ can be written. If both sides of (7) are passed to the limit when $\gamma$ goes to zero, the following equation is obtained:

$$
\tilde{G}(0, x, \lambda z) \equiv \int_{0}^{\infty} G(t, x, \lambda z) d t=E\left(\xi_{1}\right) A(x, \lambda z) .
$$

Under the conditions of the present theorem, $A(x, \lambda z)$ $=\sum_{n=0}^{\infty} \alpha_{n}(x, \lambda z) \leq E(N(\lambda z))<\infty$ for each $x>0, \mathrm{z}>0$, and $\lambda>0$. When both sides of (8) are integrated according to $\pi(z)$ from zero to infinity, the following equation is obtained:

$$
\begin{gathered}
\int_{0}^{\infty} \widetilde{G}(0, x, \lambda z) d \pi(z)=E\left(\xi_{1}\right) \int_{0}^{\infty} A(x, \lambda z) d \pi(z) \\
=E\left(\xi_{1}\right) E\left(A\left(x, \lambda \zeta_{1}\right)\right),
\end{gathered}
$$

where $E\left(\mathrm{~A}\left(\mathrm{x}, \lambda \zeta_{1}\right)\right) \equiv \int_{0}^{\infty} A(x, \lambda z) d \pi(z)$ and $\pi(z)=P\left\{\zeta_{1} \leq z\right\}, z>0$.

Substituting (9) into (5),

$$
S_{f} \equiv \frac{1}{E\left(N\left(\lambda \zeta_{1}\right)\right)} \int_{0}^{\infty} f(x) d_{x}\left(E\left(A\left(x, \lambda \zeta_{1}\right)\right)\right) .
$$

This concludes the proof of Theorem 2 .

Using Theorem 2, the exact form for the ergodic distribution function $\left(Q_{X}(x)\right)$ is given by Corollary 1 . Equation (11) can be obtained by placing an indicator function instead of $f(x)$ in (10).

Corollary 1 Assume that the assumptions of Theorem 1 are satisfied. Then, the ergodic distribution function $\left(Q_{X}(x)\right)$ of the process $X(t)$ has the following exact form:

$$
Q_{X}(x) \equiv \lim _{t \rightarrow \infty} P\{X(t) \leq x\}=\frac{E\left(A\left(x, \lambda \zeta_{1}\right)\right)}{E\left(N\left(\lambda \zeta_{1}\right)\right)},
$$

where $E\left(N\left(\lambda \zeta_{1}\right)\right)=\int_{0}^{\infty} E(N(\lambda z)) d \pi(z)$.

In addition, the characteristic function $\left(\varphi_{X}(\theta)\right)$ is given by Corollary 2 and can be obtained by placing functions $\cos (\theta x)$ and $\sin (\theta x)$ instead of $f(x)$ in (10) and, after that, by applying the Euler equation, $\exp (i \theta x) \equiv \cos$ $(\theta x)+i \sin (\theta x)$.

Corollary 2 Assume that the assumptions of Theorem 1 are satisfied. Then, the characteristic function $\left(\varphi_{X}(\theta)\right)$ of the process $X(t)$ can be expressed as follows:

$$
\varphi_{X}(\theta) \equiv \lim _{t \rightarrow \infty} E\left(e^{i \theta X(t)}\right)=\frac{E\left(A^{*}\left(\theta, \lambda \zeta_{1}\right)\right)}{E\left(N\left(\lambda \zeta_{1}\right)\right)},
$$

where

$$
E\left(A^{*}\left(\theta, \lambda \zeta_{1}\right)\right)=\int_{0}^{\infty} e^{i \theta x} d_{x}\left(E\left(A\left(x, \lambda \zeta_{1}\right)\right) .\right.
$$

Remark 1 The function $\mathrm{A}(x, \lambda z)$ is common in both the ergodic distribution function $\left(Q_{x}(x)\right)$ and characteristic function $\left(\varphi_{x}(\theta)\right)$ of the process $X(t)$. This function is difficult to obtain, even in special and easy situations. The basic identity for the random-walk process (Feller 1971, p. 600) can be used to further improve these expressions. In this context, the alternative form for the characteristic function of the ergodic distribution of the process $X(t)$ is given by Proposition 1 using the idea of Khaniyev (2003). 
Proposition 1 Assume that the assumptions of Theorem 1 are satisfied. The characteristic function $\varphi_{x}(\theta)$ of the process $X(t)$ can be expressed with characteristic functions of the boundary functional $S_{N(\lambda z)}$ and the random variable $\eta_{1}$ as follows $(\theta \in \backslash\{0\})$ :

$$
\begin{gathered}
\varphi_{X}(\theta) \equiv \lim _{t \rightarrow \infty} E\left(e^{i \theta X(t)}\right)= \\
=\frac{1}{E\left(N\left(\lambda \zeta_{1}\right)\right)} \int_{0}^{\infty} e^{i \theta \lambda z} \frac{\varphi_{S_{N(\lambda z)}}(-\theta)-1}{\varphi_{\eta}(-\theta)-1} d \pi(z),
\end{gathered}
$$

where

$$
\begin{gathered}
E\left(N\left(\lambda \zeta_{1}\right)\right)=\int_{0}^{\infty} E(N(\lambda z)) d \pi(z) ; S_{N\left(\lambda \zeta_{1}\right)}=\sum_{i=1}^{N\left(\lambda \zeta_{1}\right)} \eta_{i} \\
\varphi_{S_{N\left(\lambda \zeta_{1}\right)}}(-\theta)=E\left(e^{\left.-i \theta S_{N\left(\lambda \zeta_{1}\right)}\right) ; \varphi_{\eta}(-\theta)=E\left(e^{-i \theta \eta_{1}}\right) ;}\right. \\
\pi(z)=P\left\{\zeta_{1} \leq z\right\}, z>0 .
\end{gathered}
$$

The aim of this section is to prove the weak convergence theorem for the ergodic distribution of the standardized process $\mathrm{W}(\mathrm{t}) \equiv \mathrm{X}(t) / \lambda$, as $\lambda \rightarrow \infty$.

\section{WEAK CONVERGENCE THEOREM FOR THE ERGODIC DISTRIBUTION OF THE PROCESS $W(t)$}

The goal is to find the limit form of the ergodic distribution of the standardized process $\mathrm{W}(\mathrm{t})$, as $\lambda \rightarrow \infty$. For this reason, it is necessary to give Proposition 2 and Proposition 3. Proposition 2 Under the condition $\mu_{2} \equiv E\left(\chi_{1}^{+2}\right)<\infty$, twoterm asymptotic expansion for $M_{1}(\lambda z) \equiv E\left(S_{N(\lambda z)}\right)$ can be written as $\lambda \rightarrow \infty$ :

$$
M_{1}(\lambda z) \equiv E\left(S_{N(\lambda z)}\right)=\lambda z+\hat{\mu}_{1}+o(1)
$$

where $\hat{\mu}_{1} \equiv \frac{\mu_{2}}{2 \mu_{1}}$ and $\mu_{k}=E\left(\chi_{1}^{+k}\right), \mathrm{k}=1,2$.

Proof By using Wald identity, the following equality for $E\left(S_{N(\lambda z)}\right)$ can be written as follows:

$$
\begin{aligned}
& M_{1}(\lambda z) \equiv E\left(S_{N(\lambda z)}\right)=E\left(\sum_{i=1}^{H(\lambda z)} \chi_{i}^{+}\right)= \\
& =\mu_{1} E(H(\lambda z))=\mu_{1} U_{+}(\lambda z) .
\end{aligned}
$$

Here, $U_{+}(\lambda z)=E(H(\lambda z))$.

Asymptotic expansion for $U_{+}(\lambda z)$ is as follows (Feller 1971):

$$
U_{+}(\lambda z)=\frac{\lambda z}{\mu_{1}}+\frac{\mu_{2}}{2 \mu_{1}^{2}}+g(\lambda z)
$$

substituting (15) into (14):

$$
E\left(M_{1}\left(\lambda \zeta_{1}\right)\right)=\lambda \beta_{1}+\hat{\mu}_{1}+o(1) .
$$

Here, $\hat{\mu}_{1} \equiv \frac{\mu_{2}}{2 \mu_{1}}$ and $\mu_{k}=E\left(\chi_{1}^{+k}\right), \mathrm{k}=1,2$.

Therefore, (13) can be obtained when $\mathrm{z} \rightarrow 0$ and $\lambda \rightarrow \infty$ (see Lemma 1).

This concludes the proof of Proposition 2.

Proposition 3 Assume that the conditions $\mu_{2} \equiv \mathrm{E}\left(\chi_{1}^{+2}\right)$ and $\beta_{1} \equiv E\left(\zeta_{1}\right)<\infty$ are satisfied. Then the following asymptotic expansion can be written as $\lambda \rightarrow \infty$ :

$$
E\left(M_{1}\left(\lambda \zeta_{1}\right)\right)=\lambda \beta_{1}+\hat{\mu}_{1}+o(1) .
$$

Here, $\beta_{1} \equiv E\left(\zeta_{1}\right), \hat{\mu}_{1}=\frac{\mu_{2}}{2 \mu_{1}}$ and $\mu_{k}=E\left(\chi_{1}^{+k}\right), k=1,2$.

Proof With the help of Proposition 2, $M_{1}(\lambda z)$ can be given as follows:

$$
M_{1}(\lambda z)=\lambda z+\hat{\mu}_{1}+g(\lambda z)
$$

where $\lim _{x \rightarrow \infty} g(x)=0$

Multiplying both sides of (16) by $d \pi(\mathrm{z})$ and taking the integral from zero to infinity, one can obtain the following equality:

$$
\begin{aligned}
& E\left(M_{1}\left(\lambda \zeta_{1}\right)\right)=\int_{0}^{\infty}\left(\lambda z+\hat{\mu}_{1}+g(\lambda z)\right) d \pi(z)=\int_{0}^{\infty} \lambda z d \pi(z) \\
& +\int_{0}^{\infty} \hat{\mu}_{1} d \pi(z)+\int_{0}^{\infty} g(\lambda z) d \pi(z) .
\end{aligned}
$$

By using Lemma 1, one obtains $\int_{0}^{\infty} g(\lambda z) d \pi(z)=o(1)$. Then, asymptotic expansion for $E\left(M_{1}\left(\lambda \zeta_{1}\right)\right)$ is as follows:

$$
E\left(M_{1}\left(\lambda \zeta_{1}\right)\right)=\lambda \beta_{1}+\hat{\mu}_{1}+o(1) .
$$

Here,

$\beta_{1} \equiv E\left(\zeta_{1}\right), \hat{\mu}_{1}=\frac{\mu_{2}}{2 \mu_{1}}$ and $\mu_{k}=E\left(\chi_{1}^{+k}\right), \mathrm{k}=1,2$

This concludes the proof of Proposition 3.

By using Propositions 2 and 3, one can investigate the asymptotic behavior of the characteristic function of the ergodic distribution of the process $W(t)$.

Theorem 3 Assume that the conditions $E\left(\chi_{1}^{+2}\right)<\infty, E\left(\eta_{1}^{2}\right)$ $<+\infty$, and $E\left(\zeta_{1}\right)<\infty$ are satisfied. Two-term asymptotic expansion for the characteristic function $\varphi w(\theta)$ of the ergodic distribution of the process $W(t)$ can be written, as $\lambda \rightarrow \infty$ :

$$
\varphi_{W}(\theta)=\hat{\varphi}_{\zeta}(\theta)+\frac{1}{\lambda}\left[\hat{\varphi}_{\zeta}(\theta) D_{1}(\theta)+\frac{\hat{\mu}_{1}}{\beta_{1}}\right]+o\left(\frac{1}{\lambda}\right) .
$$

Here,

$\hat{\varphi}_{\zeta}(\theta)=\frac{\varphi_{\zeta}(\theta)-1}{i \theta \beta_{1}} ; \varphi_{\zeta}(\theta) \equiv E\left(e^{i \theta \zeta_{1}}\right) ; D_{1}(\theta)=i \theta \widehat{m}_{1}-\frac{\hat{\mu}_{1}}{\beta_{1}} ;$

$\widehat{m}_{1}=\frac{\mathrm{m}_{2}}{2 m_{1}} ; \hat{\mu}_{1}=\frac{\mu_{2}}{2 \mu_{1}} ; \mathrm{m}_{\mathrm{k}}=\mathrm{E}\left(\eta_{1}^{k}\right) ; \mu_{\mathrm{k}}=E\left(\chi_{1}^{+k}\right), k=1$, 2 and $\beta_{1}=E\left(\zeta_{1}\right)$. 
Proof An alternative form of the characteristic function of the ergodic distribution of the process $X(t)$ is as follows:

$$
\begin{gathered}
\varphi_{X}(\theta) \equiv \lim _{t \rightarrow \infty} E\left(e^{i \theta X(t)}\right)= \\
=\frac{1}{E\left(N\left(\lambda \zeta_{1}\right)\right)} \int_{0}^{\infty} e^{i \theta \lambda z} \frac{\varphi_{S_{N(\lambda z)}}(-\theta)-1}{\varphi_{\eta}(-\theta)-1} d \pi(z), \theta \neq 0 .
\end{gathered}
$$

$W(t) \equiv X(t) / \lambda$ is a linear transformation of the process $X(t)$. Then, the characteristic function of the ergodic distribution of the process $W(t)$ can be given as follows:

$$
\begin{aligned}
& \varphi_{W}(\theta) \equiv E\left(e^{i \theta W(t)}\right)=E\left(e^{i \frac{\theta}{\lambda} X(t)}\right)=\varphi_{X}\left(\frac{\theta}{\lambda}\right) \\
= & \frac{1}{E\left(N\left(\lambda \zeta_{1}\right)\right)} \int_{0}^{\infty} e^{i \theta z} \frac{\varphi_{S_{N(\lambda z)}}\left(-\frac{\theta}{\lambda}\right)-1}{\varphi_{\eta}\left(-\frac{\theta}{\lambda}\right)-1} d \pi(z), \theta \neq 0 .
\end{aligned}
$$

Divide the denominator and numerator of (17) as $I_{1}(\lambda)$ and $I_{2}(\lambda z)$, respectively:

$$
\varphi_{W}(\theta) \equiv \lim _{t \rightarrow \infty} E\left(e^{i \theta W(t)}\right)=\int_{0}^{\infty} \frac{I_{2}(\lambda z)}{I_{1}(\lambda)} d \pi(z) .
$$

Namely, $I_{1}(\lambda) \equiv E\left(N\left(\lambda \zeta_{1}\right)\right)\left[\varphi_{\eta}\left(-\frac{\theta}{\lambda}\right)-1\right]$ and

$I_{2}(\lambda z)=e^{i \theta z}\left[\varphi_{S_{N(\lambda z)}}\left(-\frac{\theta}{\lambda}\right)-1\right]$

Then, according to condition $m_{2} \equiv E\left(\eta_{1}^{2}\right)<\infty, I_{1}(\lambda)$ can be represented as follows (Feller 1971):

$$
\begin{aligned}
& I_{1}(\lambda) \equiv E\left(N\left(\lambda \zeta_{1}\right)\right)\left[\varphi_{\eta}\left(-\frac{\theta}{\lambda}\right)-1\right]=E\left(N\left(\lambda \zeta_{1}\right)\right. \\
& \left\{1-\frac{i \theta}{\lambda} m_{1}+\frac{(i \theta)^{2}}{2 \lambda^{2}} m_{2}+o\left(\frac{1}{\lambda^{2}}\right)-1\right\} \\
& =-m_{1} E\left(N\left(\lambda \zeta_{1}\right)\right) \frac{i \theta}{\lambda}\left\{1-\frac{i \theta}{2 \lambda} \frac{m_{2}}{m_{1}}+o\left(\frac{1}{\lambda}\right)\right\} .
\end{aligned}
$$

According to Wald identity, $E\left(M_{1}\left(\lambda \zeta_{1}\right)\right)=m_{1} E\left(N\left(\lambda \zeta_{1}\right)\right)$. Therefore,

$$
I_{1}(\lambda)=-E\left(M_{1}\left(\lambda \zeta_{1}\right)\right) \frac{i \theta}{\lambda}\left\{1-\frac{i \theta}{\lambda} \widehat{m}_{1}+o\left(\frac{1}{\lambda}\right)\right\}
$$

By considering the asymptotic expansion of $E\left(M_{1}\left(\lambda \zeta_{1}\right)\right)$ (see Proposition 3 ) in (19), asymptotic expansion for $I_{1}(\lambda)$ can be obtained as follows:

$$
\begin{aligned}
I_{1}(\lambda) & =-\left\{\lambda \beta_{1}+\hat{\mu}_{1}+o(1)\right\} \frac{i \theta}{\lambda}\left\{1-\frac{i \theta}{\lambda} \widehat{m}_{1}+o\left(\frac{1}{\lambda}\right)\right\} \\
& =-\left\{i \theta \beta_{1}+\frac{\theta^{2} \beta_{1}}{\lambda} \widehat{m}_{1}+\frac{i \theta}{\lambda} \hat{\mu}_{1}+o\left(\frac{1}{\lambda}\right)\right\}
\end{aligned}
$$

$$
\begin{aligned}
& =-i \theta \beta_{1}\left\{1-\frac{i \theta \widehat{m}_{1}}{\lambda}+\frac{\widehat{\mu}_{1}}{\lambda \beta_{1}}+o\left(\frac{1}{\lambda}\right)\right\}= \\
& -i \theta \beta_{1}\left\{1-\frac{1}{\lambda} D_{1}(\theta)+o\left(\frac{1}{\lambda}\right)\right\} .
\end{aligned}
$$

Here,

$$
\begin{aligned}
& D_{1}(\theta)=i \theta \widehat{m}_{1}-\frac{\hat{\mu}_{1}}{\beta_{1}} ; \hat{\mu}_{1}=\frac{\mu_{2}}{2 \mu_{1}} ; \widehat{m}_{1}=\frac{m_{2}}{2 m_{1}} ; \mu_{k}= \\
& E\left(\chi_{1}^{+k}\right) ; m_{k}=E\left(\eta_{1}^{k}\right), k=1,2 \text { and } \beta_{1}=E\left(\zeta_{1}\right) .
\end{aligned}
$$

Calculations for the numerator of (17) $I_{2}(\lambda z)$ are as follows.

$$
\begin{gathered}
I_{2}(\lambda z) \equiv e^{i \theta z}\left[\varphi_{S_{N(\lambda z)}}\left(-\frac{\theta}{\lambda}\right)-1\right]=e^{i \theta z}\left[E\left(e^{-i \frac{\theta}{\lambda} S_{N(\lambda z)}}\right)-1\right] \\
=e^{i \theta z}\left[E\left(e^{-i \frac{\theta}{\lambda}\left(\lambda z+\hat{S}_{N(\lambda z)}\right)}\right)-1\right]= \\
=e^{i \theta z}\left[\left\{e^{-i \theta z} E\left(e^{-i \frac{\theta}{\lambda} \hat{S}_{N(\lambda z)}}\right)\right\}-1\right] \\
=E\left(e^{-i \frac{\theta}{\lambda} \hat{S}_{N(\lambda z)}}\right)-e^{i \theta z}
\end{gathered}
$$

where $\hat{S}_{N(\lambda z)} \equiv S_{N(\lambda z)}-\lambda z$.

Because $\mu_{2} \equiv E\left(\chi_{1}^{+2}\right)$, the first moment of the boundary functional $\hat{S}_{N(\lambda z)}$ is finite as $\lambda \rightarrow \infty$ (Rogozin 1964). Then, one can write the Taylor expansion for the characteristic function of the boundary functional $\hat{S}_{N(\lambda z)}$ into (21) as follows:

$$
\begin{aligned}
I_{2}(\lambda z) & \equiv\left[1-i \frac{\theta}{\lambda} E\left(\widehat{S}_{N(\lambda z)}\right)+\frac{1}{\lambda} g(\lambda z)\right]-e^{i \theta z}= \\
& =1-e^{i \theta z}-i \frac{\theta}{\lambda} \hat{\mu}_{1}+\frac{1}{\lambda} g(\lambda z) .
\end{aligned}
$$

Here, $E\left(\hat{S}_{N(\lambda z)}\right)=\hat{\mu}_{1}+o(1) ; \hat{\mu}_{1}=\frac{\mu_{2}}{2 \mu_{1}}$ and $\mu_{k}=$ $E\left(\chi_{1}^{+k}\right), k=1,2$.

Multiplying both sides of (22) with $d \pi(z)$ and taking the integral from zero to infinity, one obtains

$$
\int_{0}^{\infty} I_{2}(\lambda z) d \pi(z)=\int_{0}^{\infty}\left[1-e^{i \theta z}-i \frac{\theta}{\lambda} \hat{\mu}_{1}+\frac{1}{\lambda} g(\lambda z)\right] d \pi(z)
$$$$
=\int_{0}^{\infty}\left(1-e^{i \theta z}\right) d \pi(z)-i \frac{\theta}{\lambda} \int_{0}^{\infty} \hat{\mu}_{1} d \pi(z)+
$$

$$
+\frac{1}{\lambda} \int_{0}^{\infty} g(\lambda z) d \pi(z)
$$


It is known that the last term of (23) $\int_{0}^{\infty} g(\lambda z) d \pi(\mathrm{z})=$ $o$ (1) (see Lemma 1). Then,

$$
\begin{gathered}
\int_{0}^{\infty} I_{2}(\lambda z) d \pi(z)=\int_{0}^{\infty}\left(1-e^{i \theta z}\right) d \pi(z)-i \frac{\theta}{\lambda} \hat{\mu}_{1}+o\left(\frac{1}{\lambda}\right) \\
=\left(1-\varphi_{\zeta}(\theta)\right)-i \frac{\theta}{\lambda} \hat{\mu}_{1}+o\left(\frac{1}{\lambda}\right) .
\end{gathered}
$$

Here, $\varphi_{\zeta}(\theta) \equiv E\left(e^{i \theta \zeta_{1}}\right)=\int_{0}^{\infty} e^{i \theta z} d \pi(z)$ and $\hat{\mu}_{1}=\frac{\mu_{2}}{2 \mu_{1}}$. By substituting (20) and (24) into (18), one obtains twoterm asymptotic expansion for the characteristic function of the process $W(t)$ as $\lambda \rightarrow \infty$ :

$$
\begin{gathered}
\varphi_{W}(\theta) \equiv \lim _{t \rightarrow \infty} E\left(e^{i \theta W(t)}\right)=\int_{0}^{\infty} \frac{I_{2}(\lambda z)}{I_{1}(\lambda)} d \pi(z)= \\
=\frac{\left(1-\varphi_{\zeta}(\theta)\right)-i \frac{\theta}{\lambda} \widehat{\mu}_{1}+o\left(\frac{1}{\lambda}\right)}{-i \theta \beta_{1}\left\{1-\frac{1}{\lambda} D_{1}(\theta)+o\left(\frac{1}{\lambda}\right)\right\}}= \\
=\frac{1}{-i \theta \beta_{1}}\left\{\left(1-\varphi_{\zeta}(\theta)\right)-i \frac{\theta}{\lambda} \widehat{\mu}_{1}+o\left(\frac{1}{\lambda}\right)\right\} \\
+\frac{1}{\lambda i \theta \beta_{1}}\left[\left(\varphi_{\zeta}(\theta)-1\right) D_{1}(\theta)+i \theta \hat{\mu}_{1}\right]+o\left(\frac{1}{\lambda}\right)
\end{gathered}
$$

Here,

$$
\begin{gathered}
\hat{\varphi}_{\zeta}(\theta)=\frac{\varphi_{\zeta}(\theta)-1}{i \theta \beta_{1}} ; \varphi_{\zeta}(\theta) \equiv E\left(e^{i \theta \zeta_{1}}\right) ; D_{1}(\theta)=i \theta \hat{m}_{1}-\frac{\hat{\mu}_{1}}{\beta_{1}} ; \\
\hat{m}_{1}=\frac{m_{2}}{2 m_{1}} ; \hat{\mu}_{1}=\frac{\mu_{2}}{2 \mu_{1}} \text { and } \beta_{1}=E\left(\zeta_{1}\right) .
\end{gathered}
$$

This conlodues the proof of Theorem 3 .

With the help of Theorem 3, the limit form of the characteristic function of the ergodic distribution of the process $W(t)$ can be obtained by the following theorem. Theorem 4 Assume that the conditions of Theorem 3 are satisfied. The characteristic function $\varphi_{W}(\theta)$ of the ergodic distribution of the process $W(t)$ converges to the characteristic function $\widehat{\varphi}_{\zeta}(\theta)$, as $\lambda \rightarrow \infty$, i.e.,

$$
\varphi_{W}(\theta) \underset{\lambda \rightarrow \infty}{\longrightarrow} \hat{\varphi}_{\zeta}(\theta)
$$

Here, $\hat{\varphi}_{\zeta}(\theta)=\frac{\varphi_{\zeta}(\theta)-1}{i \theta \beta_{1}} ; \varphi_{\zeta}(\theta) \equiv E\left(e^{i \theta \zeta_{1}}\right)$ and $\beta_{1}=E\left(\zeta_{1}\right)$.
Proof The asymptotic expansion of the characteristic function of the ergodic distribution of the process $W(t)$ when $\lambda \rightarrow \infty$ is obtained in Theorem 3 as follows:

$$
\varphi_{W}(\theta)=\hat{\varphi}_{\zeta}(\theta)+\frac{1}{\lambda} K(\theta)+o\left(\frac{1}{\lambda}\right) .
$$

Here, $K(\theta) \equiv \hat{\varphi}_{\zeta}(\theta) D_{1}(\theta)+\frac{\hat{\mu}_{1}}{\beta_{1}} ; D_{1}(\theta)=i \theta \hat{m}_{1}-\frac{\hat{\mu}_{1}}{\beta_{1}} ; \hat{\mu}_{1}=$ $\frac{\mu_{2}}{2 \mu_{1}}$ and $\widehat{m}_{1}=\frac{m_{2}}{2 m_{1}}$.

Consider $K(\theta)$ in (25) as follows:

$$
\begin{aligned}
|K(\theta)|= & \left|\hat{\varphi}_{\zeta}(\theta) D_{1}(\theta)+\frac{\hat{\mu}_{1}}{\beta_{1}}\right|=\left|\hat{\varphi}_{\zeta}(\theta)\left(i \theta \hat{m}_{1}-\frac{\hat{\mu}_{1}}{\beta_{1}}\right)+\frac{\hat{\mu}_{1}}{\beta_{1}}\right| \\
& \leq \theta \hat{m}_{1}+\frac{\hat{\mu}_{1}}{\beta_{1}}+\frac{\hat{\mu}_{1}}{\beta_{1}}=\theta \hat{m}_{1}+\frac{2 \hat{\mu}_{1}}{\beta_{1}} \equiv C_{1}(\theta) .
\end{aligned}
$$

Here, $C_{1}(\theta) \equiv \theta \widehat{m}_{1}+\frac{2 \widehat{\mu}_{1}}{\beta_{1}}$.

According to conditions of Theorem 3 , for each finite $\theta$, $0<\beta_{1}<\infty, m_{2}<\infty$ and $\mu_{2}<\infty$, so $C_{1}(\theta)<\infty$. In addition, $K(\theta)$ is also finite. Therefore, $K(\theta) / \lambda \rightarrow 0$ as $\lambda \rightarrow \infty$. Hence, for each finite $\theta$,

$$
\varphi_{W}(\theta) \underset{\lambda \rightarrow \infty}{\longrightarrow} \hat{\varphi}_{\zeta}(\theta),
$$

where

$\hat{\varphi}_{\zeta}(\theta)=\frac{\varphi_{\zeta}(\theta)-1}{i \theta \beta_{1}} ; \varphi_{\zeta}(\theta)=E\left(e^{i \theta \zeta_{1}}\right) ; D_{1}(\theta)=i \theta \widehat{m}_{1}-$ $\frac{\hat{\mu}_{1}}{\beta_{1}} ; \hat{\mu}_{1}=\frac{\mu_{2}}{2 \mu_{1}} ; \hat{m}_{1}=\frac{m_{2}}{2 m_{1}}$.

This concludes the proof of Theorem 4 .

Remarks 2 It is known that $\widehat{\varphi}_{\zeta}(\theta)$ is the characteristic function of the limit distribution of the residual waiting time of the renewal process generated by the sequence of random variables $\left\{\zeta_{n}\right\}, n=1,2, \ldots$ In Theorem 4 , it is shown that characteristic function of the ergodic distribution of the process $W(t)$ converges to the characteristic function $\widehat{\varphi}_{\zeta}(\theta)$, as $\lambda \rightarrow \infty$. Hence, according to the continuity theorem for characteristic functions (Feller 1971; Lukacs 1970), the ergodic distribution of the standardized process $W(t)$ weakly converges to the limit distribution of the residual waiting time of the renewal process generated by the sequence of random variables $\left\{\zeta_{n}\right\}, \mathrm{n}=1,2, \ldots$ as $\lambda \rightarrow \infty$. This statement is expressed with the help of the following theorem.

Theorem 5. Assume that the assumptions of Theorem 3 are satisfied. Then, the ergodic distribution $\left(Q_{W}(x)\right)$ of the process $W(t)$ weakly converges to the limit distribution $R(x)$, for each $x>0$, as $\lambda \rightarrow \infty$ :

$$
Q_{W}(x) \underset{\lambda \rightarrow \infty}{\longrightarrow} R(x) \equiv \frac{1}{\beta_{1}} \int_{0}^{x}(1-\pi(v)) d v .
$$

Here, $R(x)$ is the limit distribution of the residual waiting time of the renewal process generated by the 
sequence of random variables $\left\{\zeta_{n}\right\}, \mathrm{n}=1,2, \ldots$,

$$
\begin{aligned}
& Q_{W}(x) \equiv \lim _{t \rightarrow \infty} P\{W(t) \leq x\}, \beta_{1}=E\left(\zeta_{1}\right), \text { and } \pi(v)= \\
& P\left\{\zeta_{1} \leq v\right\}, v>0
\end{aligned}
$$

\section{CONCLUSION}

In this study, a generalization of the studies that exist in the literature about semi-Markovian random-walk processes with discrete interference of chance was done. This was because the discrete interference of chance in existing studies has had certain and special distributions. In the studies of Aliyev et al. (2009, 2010), Kesemen et al. (2013), and Khaniyev et al. (2008), the random variables $\zeta_{n}$ that describe the discrete interference of chance have exponential, gamma, triangular, and Weibull distribution, respectively. In some respects, what was found in this study is the generalization of the results reported in the literature. With the application of the results in this study, previous studies become a special case of this study. In summary, a semi-Markovian random-walk process with general interference of chance was considered, and its mathematical model was constructed. The ergodicity of the process $X(t)$ was proved, and the exact expression of the characteristic function of the ergodic distribution $X$ $(t)$ was obtained. Then, two-term asymptotic expansion for the characteristic function of ergodic distribution $\left(\varphi_{W}(\theta)\right)$ of the standardized process $W(t) \equiv X(t) / \lambda$ was given. It was shown that the characteristic function of the ergodic distribution of the process $W(t)$ converges to the characteristic function of the limit distribution of the residual waiting time of the renewal process generated by random variable $\zeta_{n}$ describing the discrete interference of chance. As a result, it was stated that the ergodic distribution of the standardized process $W(t)$ weakly converges to the limit distribution of the residual waiting time of the renewal process generated by random variable $\zeta_{n}$. In future studies, it is planned to investigate the case where discrete interference of chance belongs to a wider class. In addition, it is planned to obtain three-term asymptotic expansions for the stationary characteristics of the process $X(t)$.

\section{ACKNOWLEDGEMENTS}

The authors would like to thank the anonymous referees for their helpful and constructive comments that greatly contributed to this paper. We would also like to thank the editor for her generous comments and support during the review process.

\section{REFERENCES}

Aliyev, R., Ardic, O. \& Khaniyev, T. 2016. Asymptotic approach for a renewal-reward process with a general interference of chance. Communication in Statistics-Theory and Methods 45(14): 4237-4248
Aliyev, R., Khaniyev, T. \& Kesemen, T. 2009. Asymptotic expansions for the moments of the semi-Markovian random walk with Gamma distributed interference of chance. Communication in Statistics-Theory and Methods 39(1): 130-143.

Aliyev, R., Kucuk, Z. \& Khaniyev, T. 2010. Three-term asymptotic expansions for the moments of the random walk with triangular distributed interference of chance. Applied Mathematical Modelling 34(11): 3599-3607.

Alsmeyer, G. 1991. Some relations between harmonic renewal measure and certain first passage times. Statistics and Probability Letters 12(1): 19-27.

Başar, E. 2017. Aalen's additive, Cox proportional hazards and the Cox-Aalen model: Application to kidney transplant data. Sains Malaysiana 46(3): 469-476.

Blanchet, J. \& Glynn, P. 2006. Complete corrected diffusion approximations for the maximum of a random walk. The Annals of Applied Probability 16(2): 951-983.

Brown, M. \& Solomon, H.A. 1975. Second-order approximation for the variance of a renewal-reward process. Stochastic Processes and Their Applications 3(3): 301-314.

Chang, J.T. \& Peres, Y. 1997. Ladder heights, Gaussian random walks and the Riemann zeta function. Annals of Probability 25(2): 787-802.

Feller, W. 1971. Introduction to Probability Theory and Its Applications II. New York: John Wiley.

Gihman, I.I. \& Skorohod, A.V. 1975. Theory of Stochastic Processes II. Berlin: Springer.

Gökpınar, E., Khaniyev, T. \& Gamgam, H. 2015. Asymptotic properties of the straight line estimator for a renewal function. Sains Malaysiana 44(7): 1041-1051.

Gökpınar, F., Khaniyev, T. \& Mammadova, Z. 2013. The weak convergence theorem for the distribution of the maximum of a Gaussian random walk and approximation formulas for its moments. Methodology and Computing in Applied Probability 15: 333-347.

Hanalioglu, Z. \& Khaniyev, T. 2019. Limit theorem for a semiMarkovian stochastic model of type (s,S). Hacettepe Journal of Mathematics and Statistics 48(2): 605-615.

Hanalioglu, Z., Khaniyev, T. \& Agakishiyev, I. 2015. Weak convergence theorem for the ergodic distribution of a random walk with normal distributed interference of chance. TWMS Journal of Applied and Engineering Mathematics 5(1): 61-73.

Janseen, A.J.E.M. \& Van Leeuwarden, J.S.H. 2007. On Lerch's transcendent and the Gaussian random walk. Annals of Applied Probability 17(2): 421-439.

Kesemen, T., Aliyev, R. \& Khaniyev, T. 2013. Limit distribution for a semi-Markovian random walk with Weibull distributed interference of chance. Journal of Inequalities and Applications doi.org/10.1186/1029-242X-2013-134.

Khaniyev, T. \& Mammadova, Z. 2006. On the stationary characteristics of the extended model of type $(\mathrm{s}, \mathrm{S})$ with Gaussian distribution of summands. Journal of Statistical Computation and Simulation 76(10): 861-874.

Khaniyev, T. 2003. Some asymptotic results for the semiMarkovian random walk with a special barrier. Turkish Journal of Mathematics 27: 251-272.

Khaniyev, T., Kesemen, T., Aliyev, R. \& Kokangul, A. 2008. Asymptotic expansions for the moments of a semiMarkovian random walk with exponential distributed interference of chance. Statistics and Probability Letters 78(6): 785-793. 
Lotov, V.I. 1996. On some boundary crossing problems for Gaussian random walks. Annals of Probability 24(4): 2154-2171.

Lukacs, E. 1970. Characteristic Functions. London: Griffin.

Nagaev, S.V. 2010. Exact expressions for the moments of ladder heights. Siberian Mathematical Journal 51(4): 675-695.

Rogozin, B.A. 1964. On the distribution of the first jump. Theory of Probability and Its Applications 9(3): 450-465.

Samsuddin, S. \& Ismail, N. 2019. Markov chain model and stationary test: A case study on Malaysia Social Security (SOCSO). Sains Malaysiana 48(3): 697-701.

Siegmund, D. 1979. Corrected diffusion approximations in certain random walk problems. Advances in Applied Probability 11(4): 701-719.

Tahir Khaniyev \& Ozlem Ardic Sevinc* Department of Industrial Engineering TOBB University of Economics and Technology 06560, Ankara

Turkey
Ozlem Ardic Sevinc*

Department of Structural Economic Research Central Bank of the Republic of Turkey 06050, Altindag, Ankara

Turkey

Tahir Khaniyev

Institute of Control Systems

Azerbaijan National Academy of Sciences

AZ 1141, Baku

Azerbaijan

*Corresponding author; email: ardicozlem@gmail.com

Received: 29 June 2019

Accepted: 6 December 2019 